\title{
Transfer of a Cytoplasmic Factor by Micro-injection in Verticillium
}

\author{
By MILTON A. TYPAS \\ Biology Department, University of Athens, Panepistemiopolis, \\ Kouponia TT 621, Athens, Greece \\ AND JAMES B. HEALE \\ Biology Department, Queen Elizabeth College, London $W 87 A H$
}

(Received 7 August 1978)

\begin{abstract}
Preparations of protoplasm, nuclei and mitochondria were introduced into living Verticillium cells. Most of the hyphae of auxotrophs injected with protoplasm obtained from complementary auxotrophs survived micro-injection ( $80 \%$ ). Of these, $21 \%$ formed 'artificial' heterokaryons from which both parental genotypes were recovered by conidial analysis. Reciprocal injections of protoplasm were made between auxotrophic strains with darkly-pigmented resting structures $\left(\mathrm{Hyl}^{+}\right)$and hyaline strains lacking resting structures $(\mathrm{Hyl})$. Conidial analysis resulted in the recovery of all auxotrophic markers. The majority of phenotypes having darkly-pigmented resting structures were of the $\mathrm{Hyl}^{+}$type (92 to $96 \%$ ), but a few were partially or completely hyaline. Reciprocal injections between $\mathrm{Hyl}^{+}$or $\mathrm{Hyl}^{+}$' sooty' (Sot) strains and $\mathrm{Hyl}$ or $\mathrm{Hyl} \mathrm{Sot}^{+}$strains (hyaline strains induced by ultravioletirradiation from Sot auxotrophs) revealed the cytoplasmic inheritance of the Hyl marker in contrast to the nuclear inheritance of the Sot marker. The survival of recipient cells injected with preparations of nuclei was 30 to $40 \%$ on complete medium but conidial analyses indicated that heterokaryons were not produced. The survival of hyphae injected with mitochondrial preparations was 40 to $45 \%$, and these colonies showed considerable morphological instability which was overcome during 2 to 3 weeks incubation. Conidial analyses of $\mathrm{Hyl}$ recipients injected with $\mathrm{Hyl}^{+}$mitochondrial preparations resulted in 13 to $21 \%$ darkly-pigmented variants. These results suggest that one or more factors controlling darkly-pigmented structures were present in the mitochondrial preparations from the $\mathrm{Hyl}^{+}$ donor cytoplasm.
\end{abstract}

\section{INTRODUCTION}

Although micromanipulation has attracted interest since Barber (1911) developed his micro-injection apparatus, there are relatively few organisms in which transplantation has been attempted. Most research has involved the transplantation of different substances into protozoan cells, e.g. Amoeba (Comandon \& de Fonbrune, 1939; Kopac, 1959), Paramecium aurelia (Gibson, 1973; Koizumi, 1974; Knowles, 1974) and amphibian eggs (Briggs \& King, 1959). Burgeff (1914) was the first to transplant fungal nuclei using Phycomyces nitens; more recently, Wilson (1961, 1963, 1969), Wilson et al. (1961) and Diakumacos et al. (1965) have reported the successful transplantation of functional nuclei in Neurospora crassa.

The frequency with which black parental cultures of the asexual fungi Verticillium alboatrum and Verticillium dahliae become hyaline, or produce hyaline sectors after repeated transfer in culture, has been studied by Isaac (1949), Pegg (1957), Heale \& Isaac (1964) and 
Brandt \& Roth (1965). The wild-type isolates of these fungi form a dark pigment after 5 to $10 \mathrm{~d}$, which is deposited in the walls of the resting structures (resting mycelium of $V$. albo-atrum and microsclerotia of $V$. dahliae). The inheritance of dark resting structures has been attributed to various mechanisms including heterokaryosis and the dual phenomenon (Hansen, 1938), genetic recombination (Robinson et al., 1957), the parasexual cycle (Hastie, 1964), changes in ploidy levels (Tolmsoff, 1972, 1973) and, more recently, to cytoplasmic inheritance (Typas, 1976; Typas \& Heale, 1976 a, b, 1978). The most convenient method for the detection of cytoplasmic determinants is the heterokaryon test devised by Jinks (1964). In Verticillium, heterokaryons are formed by the fusion of hyphal tips or germ-tubes.

The important role played by cytoplasmic factors in the inheritance of darkly-pigmented resting structures was indicated by intensive heterokaryon analyses (Typas \& Heale, 1976b). We have now studied the transfer of protoplasm, viable nuclei and mitochondrial fractions from a darkly-pigmented donor to a hyaline recipient, and vice versa, in an attempt to investigate the genetics of these fungi.

\section{METHODS}

Before micro-injection experiments could be undertaken, it was necessary to ensure that compatible protoplasm could be injected without harm to the recipient cell. Auxotrophic strains which were compatible when 'forced' to form heterokaryons on minimal medium (MM) were therefore used (Typas \& Heale, 1976b).

Equipment. A modification of Wilson's technique (1961) was used. The microneedle was connected by vinyl tubing to a syringe $(50 \mu \mathrm{l})$ controlled by a micrometer screw (Fig. 1). The plastic slide oil-chamber of Holwill \& McGregor (1974) was used. Observations were made using a Meopta D microscope mounted on a Research Instruments TVC 500 Bio-Medical micromanipulator, using $\times 45$ or $\times 100$ microscope objectives and a calibrated eye-piece micrometer.

Preparation of microtools. Microneedles were first pulled in a Nasishige (NE-2) vertical micro-electrodepulling machine and further extended and shaped in a de Fonbrune (MV) microforge. The final diameter of a microneedle tip was between 0.5 and $1.0 \mu \mathrm{m}$. Tubes with an internal diameter larger than $2 \mu \mathrm{m}$ were used as micropipettes. From a large number of microneedles produced, only those with a sharp tip like that of a hypodermic needle were used for micro-injections; the rest were used as scalpels and hooks. When micropipettes with large internal diameters were needed for collecting the mitochondrial preparations, the tips were broken off by pushing them against a solid surface.

Microchambers were individually sterilized on filter paper in glass Petri dishes. The chambers were covered with coverslips sterilized by autoclaving between layers of filter paper in a glass Petri dish.

Microcultures. Microcultures of the auxotrophic strains listed in Table 1 were prepared by the method of Wilson (1961) with minor modifications. Hanging-drop preparations of Verticillium hyphae were only useful when acting as the donor culture whereas agar films were equally useful as donor or recipient cultures. A hanging-drop was placed on the underside of a coverslip and mounted in a chamber, the size of the drop being controlled by means of a micropipette; by reducing the drop diameter, cells could be induced to adhere to the coverslip.

Agar films were prepared by covering one surface of sterile $24 \times 30 \mathrm{~mm}$ no. 2 glass coverslips with a thin layer of MM supplemented with the necessary nutritional requirements of the auxotrophic mutants used. The coverslips were placed, film side up, in a sterile Petri dish containing sterile filter paper and were then inoculated with small agar blocks cut from the edge of actively growing mycelium on complete medium (CM; Typas \& Heale, 1976b). The inocula were placed upside-down on the agar films, and the filter paper was moistened with sterile water. The microcultures were incubated at $23{ }^{\circ} \mathrm{C}$ until hyphae had grown about $1 \mathrm{~cm}$ and then the agar block inocula were removed. The microcultures were kept at about $4{ }^{\circ} \mathrm{C}$ until used. Such cultures were started 2 to $4 \mathrm{~d}$ before micro-injection. Inoculation of agar films with colonies started from mycelium instead of conidia ensured that growth consisted of relatively few, well-separated large hyphae as this was most suitable for the cutting and removal of sections.

Plasmolysing solutions were prepared using $10 \mathrm{ml}$ samples of sterile sucrose solutions ranging from 10 to $25 \%(\mathrm{w} / \mathrm{v})$. These solutions reduced the internal pressure of the hyphae so that the wall could be punctured by the microneedle without loss of cytoplasm.

Micro-injection procedures. The techniques outlined by Wilson $(1961,1963)$, Wilson et al. (1961) and Diakumacos et al. (1965) were used with minor modifications. Vegetative hyphae of Verticillium growing 
(b)

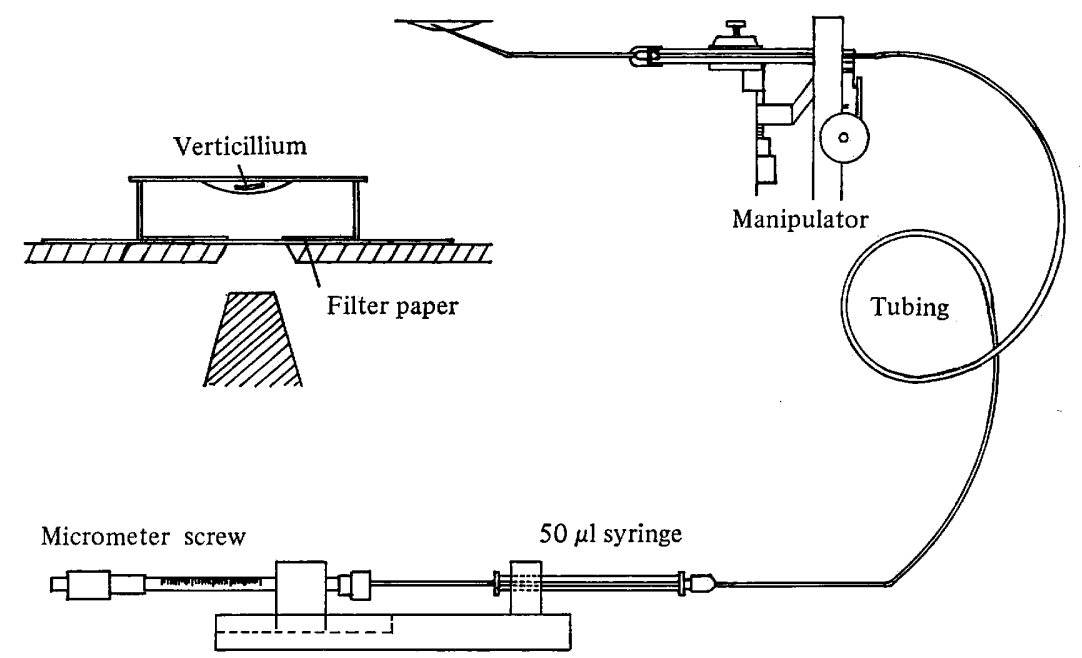

(a)

Fig. 1. Diagram of micromanipulation equipment: $(a)$ whole system; (b) moist chamber.

Table 1. Auxotrophic strains of Verticillium used Pigmented strains $\left(\mathrm{Hyl}^{+}\right)$

\begin{tabular}{|c|c|c|c|c|c|}
\hline & \multicolumn{3}{|c|}{ 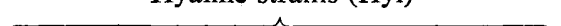 } \\
\hline $\begin{array}{l}\text { Strain } \\
\text { desig- } \\
\text { nation }\end{array}$ & $\begin{array}{c}\text { Resting } \\
\text { structure } \\
\text { phenotype* }\end{array}$ & $\begin{array}{c}\text { Auxotrophic } \\
\text { genotype }\end{array}$ & $\begin{array}{l}\text { Strain } \\
\text { desig- } \\
\text { nation }\end{array}$ & $\begin{array}{c}\text { Resting } \\
\text { structure } \\
\text { phenotype* }\end{array}$ & $\begin{array}{l}\text { Auxotrophic } \\
\text { genotype }\end{array}$ \\
\hline \multicolumn{6}{|c|}{ Verticillium albo-atrum } \\
\hline$a$ & $\mathrm{Hyl}^{+} 1$ & met, nic, trp & $d$ & Hyl Sot ${ }^{+} 6$ & ino- 4, sot $-6^{+}$ \\
\hline$b$ & $\mathrm{Hyl}^{+}$Sot & arg-9, sot -1 & $e$ & Hyl 1 & ade- 4 , arg-4 \\
\hline$c$ & $\mathrm{Hyl}^{+} 2$ & arg-10, cho-4 & $f$ & Hyl 1 & met-3, nic-3 \\
\hline \multicolumn{6}{|c|}{ Verticillium dahliae } \\
\hline$g$ & $\mathrm{Hyl}^{+} \mathbf{1}$ & ade-5, bio-1 & $j$ & Hyl 1 & arg-7, pro-1 \\
\hline$h$ & $\mathrm{Hyl}^{+} 1$ & nic, lys & $k$ & Hyl 1 & ade-8, gua-1 \\
\hline $\mathrm{i}$ & $\mathrm{Hyl}^{+} 2$ & asp, ino & $l$ & Hyl 1 & met-7, ane-4 \\
\hline
\end{tabular}

* Phenotype definitions: $\mathrm{Hyl}^{+}$, wild-type phenotype producing normal amounts of darkly-pigmented resting structures; $\mathrm{Hyl}$, hyaline strains never producing dark pigment or resting structures, obtained from $\mathrm{Hyl}^{+}$wild-type by ultraviolet-irradiation or acriflavine treatment; $\mathrm{Hyl}^{+}$Sot, 'sooty' mutants of $V$. albo-atrum kindly provided by Dr A. C. Hastie, Dundee University, they are distinct from the wild-type in that their black pigmentation extends to the edge of the culture after only 4 to $5 \mathrm{~d}$ growth at $23^{\circ} \mathrm{C}$; Hyl Sot ${ }^{+}$, hyaline strains obtained from 'sooty' strains by ultraviolet-irradiation.

on agar films have a small diameter ( 2 to $4 \mu \mathrm{m}$ ) compared with the $15 \mu \mathrm{m}$ diameter hyphae of Neurospora. The hyphae of Verticillium have septa at fairly regular intervals, and each septum has a pore in the centre through which the cytoplasm may flow. The donor microculture was inverted over a microchamber filled with silicone oil and anchored in place with sterile vaseline. The microneedle was filled with cytoplasm and nuclei by inserting the needle into several cells and removing their contents. The tip of the microneedle was kept immersed in silicone oil to prevent exposure to air. A sterile microchamber was filled with $10 \%$ sucrose solution and the microculture to be used as a recipient was inverted over the microchamber and anchored with vaseline. During the exchange of microchambers the tip of the microneedle was exposed to air, but the protoplasmic fluid was protected by the cap of silicone oil. Just before the microneedle was inserted into the recipient hypha, the oil ahead of the fluid was ejected. Injections of donor protoplasm into the recipient hyphae were made indirectly by piercing a cell next to the septum and making the injection through the septal pore leading in to the next cell. The micrometer syringe was turned slowly and the fluid for injection was expelled into the recipient cell. The injection was usually successful if the treated cell was sealed off by a plug with little or no leakage. The most suitable part of the hypha for injection was the region starting 1 to 2 cells from the tip and extending back for approximately 
30 to 40 cells. These cells formed plugs in approximately 50 to $60 \mathrm{~s}$ and this was important for their survival. The microneedle was not pulled away before a plug had been formed, and only small amounts (never more than one-tenth of the cell volume) of cytoplasm and nuclei were injected to prevent bursting of the recipient cell. A hyphal fragment of 3 to 4 cells, including the injected cell, was cut out by means of a micropipette or microscalpel and transferred with a micropipette to a marked area of a Petri plate containing CM. The uninjected cells in the fragment were previously destroyed by puncturing, and then filled with silicone oil to serve as markers. After $48 \mathrm{~h}$ incubation at $23^{\circ} \mathrm{C}$ in the dark, the injected fragments were examined under the microscope and those growing were transferred to $\mathrm{MM}$ plates with a small $\mathrm{CM}$ agar block to initiate growth. After 2 to 4 weeks incubation, conidial analysis was carried out by plating spores on CM plates followed by replica plating on appropriately supplemented MM. Using the same technique, one series of cells was injected with a sterile $10 \%(\mathrm{w} / \mathrm{v})$ sucrose solution and other cells were punctured but not injected; these were kept as controls. Because of the small size of the nuclei (about $1.0 \mu \mathrm{m}$ diam.) and the low magnification used it was not possible to follow the injection of nuclei visually. Successful nuclear transfer could however, be inferred genetically by changes in the recipient genotype. The degree of sensitivity of cells of the same hypha to the insertion of a microneedle varied greatly. In preliminary experiments some cells died instantly by losing their protoplasmic contents, some died within 1 to $2 \mathrm{~min}$ and others were unharmed although the microneedle was inserted and kept in the cell for 7 to $8 \mathrm{~min}$. Only when the microneedle was inserted slowly into the cell did the protoplasmic membrane remain undamaged with a higher number of survivors being obtained.

\section{Preparation of injection fluids}

Isolation of protoplasm, cytoplasm and nuclei. Protoplasmic fluid was obtained by inserting a microneedle into several cells of the donor hyphae and removing their contents. It was almost immediately injected into the recipient cell and the micro-injection operations never exceeded 90 min duration using the same fluid.

Isolation of nuclei. Nuclear preparations were made from different auxotrophs as well as from wild-type strains (Table 1) by the method of Reich \& Tsuda (1961). Strains were incubated in stationary CM liquid medium at $23{ }^{\circ} \mathrm{C}$. After 7 to $10 \mathrm{~d}$ growth the mycelium was washed well with sterile distilled water and pressed between sheets of sterile filter paper until no further moisture could be removed. The resultant mycelial pellet was ground in a mortar at $4{ }^{\circ} \mathrm{C}$ with three times its weight of sterile washed sand until a homogeneous mass had been obtained. Three volumes of $0.35 \mathrm{M}$-mannitol were added and the paste was stirred until smooth. The resulting thick suspension was poured into a large funnel lined with three layers of cheesecloth and, after $40 \mathrm{~min}$, an opaque orange fluid was recovered as filtrate which was almost completely free of mycelial fragments and sand. This homogenate was used for the isolation of both mitochondria and nuclei.

The crude homogenate was centrifuged at $500 \mathrm{~g}$ for $5 \mathrm{~min}$. Two layers were visible in the sedimented pellet: an outer white zone was discarded, and an opaque gelatinous central layer was resuspended in the supernatant and centrifuged again at $2000 \mathrm{~g}$ for $7 \mathrm{~min}$. This yielded a larger pellet whose appearance resembled that obtained at $500 \mathrm{~g}$. The central and superficial portions were resuspended in $0.35 \mathrm{M}$-mannitol and combined with the pellet obtained from centrifuging the supernatant for a further $30 \mathrm{~min}$ at $5000 \mathrm{~g}$. The resulting suspension was examined by electron microscopy and found to be rich in nuclei, most of which could be sedimented in relatively pure form by centrifugation at $3200 \mathrm{~g}$ for $7 \mathrm{~min}$. The resulting pellet, resuspended in an equal volume of supernatant, served as the injection fluid.

The amount of nuclear suspension injected probably ensured that at least one nucleus of the donor strain was injected into the cells during each operation. If viable nuclei from a donor auxotroph were successfully injected into a complementary auxotrophic mutant, an 'artificial' heterokaryon would be formed which could then be detected by its growth on MM.

Isolation of mitochondria. Mitochondrial preparations were obtained by isopycnic centrifugation in a continuous sucrose density gradient. A homogenate was prepared as described above, and then centrifuged at $500 \mathrm{~g}$ for $10 \mathrm{~min}$. One $\mathrm{ml}$ of the supernatant, free of nuclei and debris after the preliminary centrifugation and containing the equivalent of about $0.2 \mathrm{~g}$ wet wt of mycelium, was layered on a $4 \mathrm{ml}$ continuous density gradient prepared according to the method of Luck (1963), using 0.58 and $1.9 \mathrm{M}$-sucrose in $1 \mathrm{mM}$-EDTA. Centrifugation was carried out for $4 \mathrm{~h}$ at $38000 \mathrm{rev}$. $\mathrm{min}^{-1}$ in an MSE Superspeed centrifuge. After centrifugation, the gradients were kept at $5{ }^{\circ} \mathrm{C}$ until the highly dense mitochondrial bands became visible; the bands were collected using a sterile micropipette. The mitochondrial preparations were kept at $4{ }^{\circ} \mathrm{C}$ and were used for micro-injections within $36 \mathrm{~h}$. 
RESULTS

\section{Micro-injection of protoplasm}

Protoplasmic fluid was injected into different recipient cells, and if the cells became sealed off with little or no leakage of cytoplasm or injected material, the injection was considered successful regardless of their subsequent growth. Prototrophic growth of the injected recipient on $\mathrm{MM}$, and recovery of donor auxotrophic genotypes among the first conidial generation, indicated that viable nuclei had been transferred resulting in an 'artificial' heterokaryon. Failure of the recipient cell to grow on MM could have been due to the transplantation of cytoplasm alone or of non-viable nuclei.

The strains in Table 1 were injected with protoplasm and the results are given in Table 2. Some pairs of strains were reciprocally injected using $\mathrm{Hyl}^{+}$(darkly-pigmented) and $\mathrm{Hyl}$ (completely hyaline) auxotrophs, e.g. $a \times d, d \times a, c \times f, f \times c$ (Table 2); other combinations involved $\mathrm{Hyl}$ as recipients only, e.g. $a \times e, b \times f$, and a few involved Hyl protoplasmic injections into $\mathrm{Hyl}$ recipients, e.g. $j \times k, k \times l$. From 165 successfully injected cells, 132 survived $(80 \%)$ and showed some growth after 2 to $3 \mathrm{~d}$ incubation on CM plates. Of the 132 surviving recipients on CM, 28 grew on MM, indicating that viable donor nuclei were transferred in about $21 \%$ of these injections. Three successfully injected recipients from each strain were used in control tests: for all strains, a total of 45 out of 48 survived puncturing alone; similarly, 39 out of 48 survived injection with $10 \%$ sucrose. The survival after injection with protoplasm or $10 \%$ sucrose solution was somewhat lower $(80 \%$ and $75.4 \%$, respectively) than that for punctured and uninjected cells $(92.0 \%)$. The lag period before a distinct mycelial front was established in the heterokaryons was always greater than that in 'natural' forced heterokaryons (Typas \& Heale, 1976b). Once established, most of the 'artificial' heterokaryons grew more slowly than the 'natural' heterokaryons, but a few grew at the same rate. Sporulation after 6 weeks incubation was sparse; colonies from $\mathrm{Hyl}^{+} / \mathrm{Hyl}^{+}$or $\mathrm{Hyl}^{+} / \mathrm{Hyl}$ heterokaryons yielded $3 \times 10^{5}$ to $7 \times 10^{5}$ conidia per plate whereas the 'natural' heterokaryons produced at least 10 times greater yields of conidia. As in 'natural' heterokaryons, the $\mathrm{Hyl} / \mathrm{Hyl}$ combinations always produced 10 to 70 times fewer conidia than the corresponding $\mathrm{Hyl}^{+} / \mathrm{Hyl}^{+}$darkly-pigmented heterokaryons. 'Artificial' heterokaryons which were formed by the injection of $\mathrm{Hyl}^{+}$into $\mathrm{Hyl}$ recipients or vice versa usually showed wild-type or near wild-type characteristics with reduced amounts of dark pigmentation, similar to that of partially hyaline mutants. 'Artificial' heterokaryons between different combinations of $\mathrm{Hyl}$ auxotrophs never produced dark pigmentation (Table 3).

Conidial analysis of the first generation of spores formed by the 28 heterokaryotic cultures (Table 2) was carried out after 5 to 6 weeks incubation, and the genotypes and phenotypes of the resultant colonies were determined (Table 4). In all strains both of the auxotrophic markers of the parental types were recovered as expected for segregating nuclear markers in heterokaryons (varying from $1: 1$ to $1: 8$ ratios). But pigment formation varied greatly, with most of the colonies producing some dark pigmentation. Subculturing of these heterokaryotic colonies on CM and examination of the next two spore generations revealed no major changes in morphology or pigmentation.

Conidial analysis of 'artificial' heterokaryons between $\mathrm{Hyl}^{+}$Sot (darkly-pigmented, 'sooty' strains) and Hyl Sot ${ }^{+}$(completely hyaline strains derived from 'sooty' strains by ultraviolet-irradiation) auxotrophs resulted mainly in darkly-pigmented colonies in the first spore generation. Colonies with the $\mathrm{Hyl}^{+}$Sot donor auxotrophic genotype (i.e. arg-9, sot-1) were also 'sooty' ( $\left.\mathrm{Hyl}^{+} \mathrm{Sot}\right)$ in morphology, but the pigmented colonies of the recipient auxotrophic genotype were of $\mathrm{Hyl}^{+} \mathrm{Sot}^{+}$morphology. Although nutritional and 'sooty' nuclear markers were recovered as expected, colonies with the Hyl auxotrophic genotype had acquired $\mathrm{Hyl}^{+}$-type dark pigmentation. When the latter colonies were examined for two further generations, they retained the $\mathrm{Hyl}^{+}$pigmented character. 


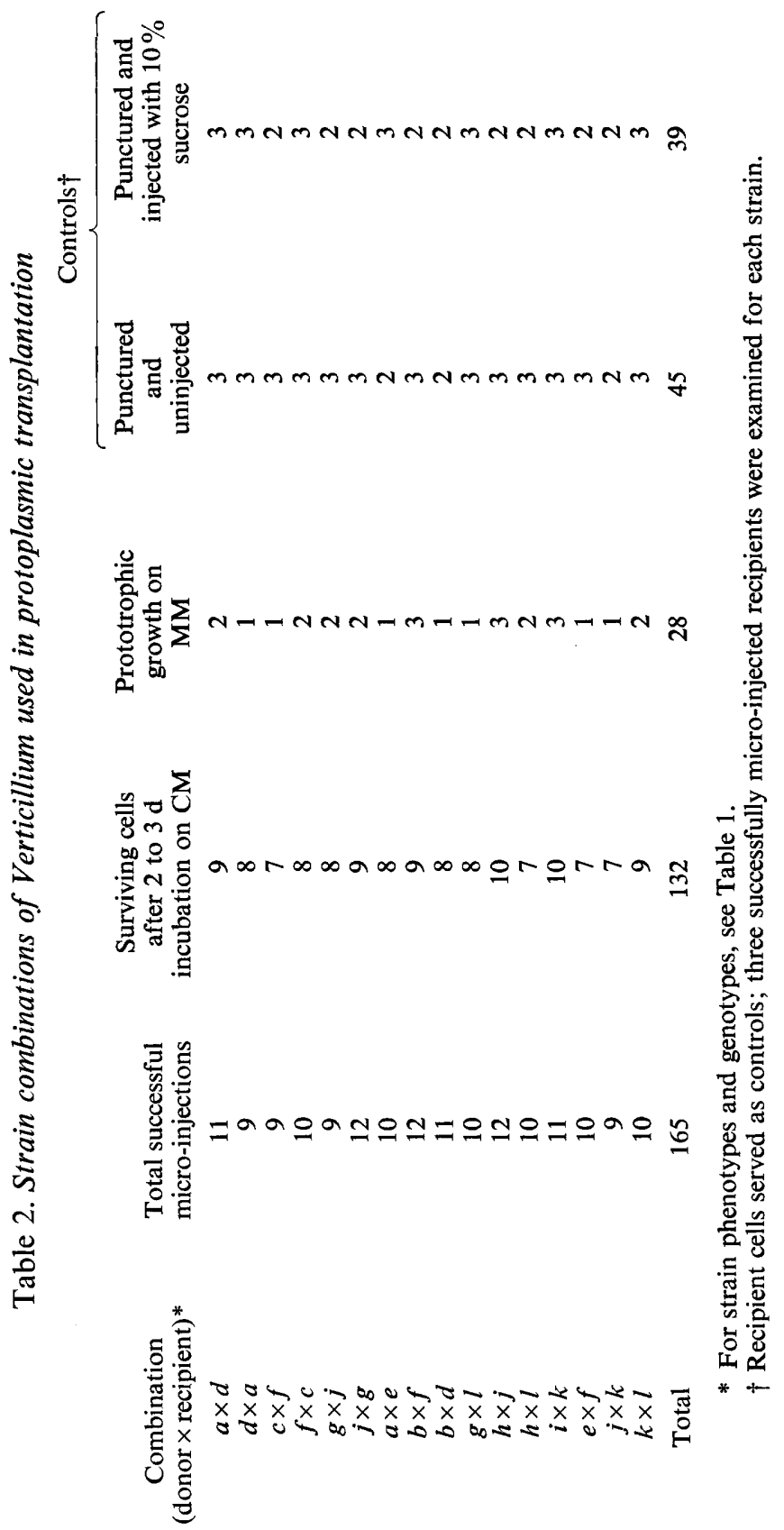


Table 3. 'Artificial' heterokaryons formed by micro-injection of protoplasm from one donor auxotrophic strain of Verticillium to the cells of another complementing recipient auxotrophic strain, and their phenotype with respect to formation of darkly-pigmented resting structures

$\begin{array}{cc}\text { Combination } & \text { Resting structure } \\ \text { (donor } \times \text { recipient) } & \text { phenotype of heterokaryons } \\ a \times d & \text { Wild-type } \\ d \times a & \text { Wild-type } \\ c \times f & \text { Wild-type } \\ f \times c & \text { Wild-type } \\ g \times j & \text { Almost wild-type } \\ j \times g & \text { Wild-type } \\ a \times e & \text { Partially hyaline } \\ b \times f & \text { Almost wild-type } \\ b \times d & \text { Almost wild-type } \\ g \times l & \text { Partially hyaline } \\ h \times j & \text { Almost wild-type } \\ h \times l & \text { Wild-type } \\ i \times k & \text { Almost wild-type } \\ e \times f & \text { Hyaline } \\ j \times k & \text { Hyaline } \\ f \times l & \text { Hyaline } \\ * \text { For strain phenotypes and genotypes, see Table } 1 .\end{array}$

Conidial analysis of heterokaryons between $\mathrm{Hyl}^{+}$Sot and $\mathrm{Hyl}$ Sot auxotrophs gave similar results. All darkly-pigmented colonies with $\mathrm{Hyl}^{+}$Sot or $\mathrm{Hyl} \mathrm{Sot} \mathrm{auxotrophic} \mathrm{geno-}$ type were of the 'sooty' pigmented type. Tests between Hyl and Hyl auxotrophs led to the recovery of only parental types of both auxotrophic and morphological characteristics.

Failure to transplant viable donor nuclei with the protoplasmic fluid into the recipient cells could still result in transfer of donor cytoplasm (and therefore also the Hyl factor) even in the absence of prototrophic growth. When the recipients which survived injection but which failed to grow on MM after 7 to $10 \mathrm{~d}$ incubation were replaced on CM plates, 74 out of 105 grew. Conidial analysis of these colonies revealed the genotypes of the first generation of spores (Table 5). Only 6 out of 74 surviving strains produced conidia of both donor and recipient genotypes. These included two derived from heterokaryon combination $c \times f$ and one from each of the combinations $g \times j, b \times f, g \times l, j \times k$; from the rest only recipient auxotroph genotypes were recovered as expected. Most of the colonies from such nonprototrophs involving injection of $\mathrm{Hyl}$ auxotrophs into $\mathrm{Hyl}^{+}$recipients were wild-type or near wild-type in morphology $(95.2$ to $96.6 \%)$. In comparison with the results from conidial analysis of the corresponding 'natural' heterokaryons (Typas \& Heale, 1976b), more partially hyaline colonies (3.4 to $4.8 \%$ ) were recovered. Conidial analysis of Hyl recipients injected with $\mathrm{Hyl}^{+}$cytoplasm resulted mainly in pigmented colonies (wild-type or near wild-type pigmentation, 87.0 to $90.4 \%$ ), but the percentage of partially hyaline variants was higher $(8.0$ to $11.2 \%)$ than above and small numbers of completely hyaline colonies were also recovered (1.6 to $1.9 \%$ ). When $\mathrm{Hyl} \mathrm{Sot}{ }^{+}$recipients were injected with $\mathrm{Hyl}^{+}$Sot cytoplasm all the darkly-pigmented colonies recovered from the conidial analysis were of the $\mathrm{Hyl}^{+} \mathrm{Sot}^{+}$genotype. Finally, conidial analysis of Hyl recipients injected with $\mathrm{Hyl}$ cytoplasm always resulted in the recovery of only hyaline recipient genotypes and phenotypes. Thus, the recovery of $\mathrm{Hyl}^{+}$colonies from $\mathrm{Hyl}$ recipients which had been injected with $\mathrm{Hyl}^{+}$or $\mathrm{Hyl}^{+}$Sot cytoplasm, but not nuclei (as judged by prototrophic growth), again illustrated the extrachromosomal nature of the factor controlling the inheritance of darklypigmented resting structures in these fungi. 
Table 4. Analysis of conidia from the entire surface of 'artificial' heterokaryons of Verticillium

Conidia were classified according to their phenotypes with respect to darkly-pigmented resting structures, and the results show the percentages in each pigmentation class. At least 400 conidia were tested for each heterokaryon.

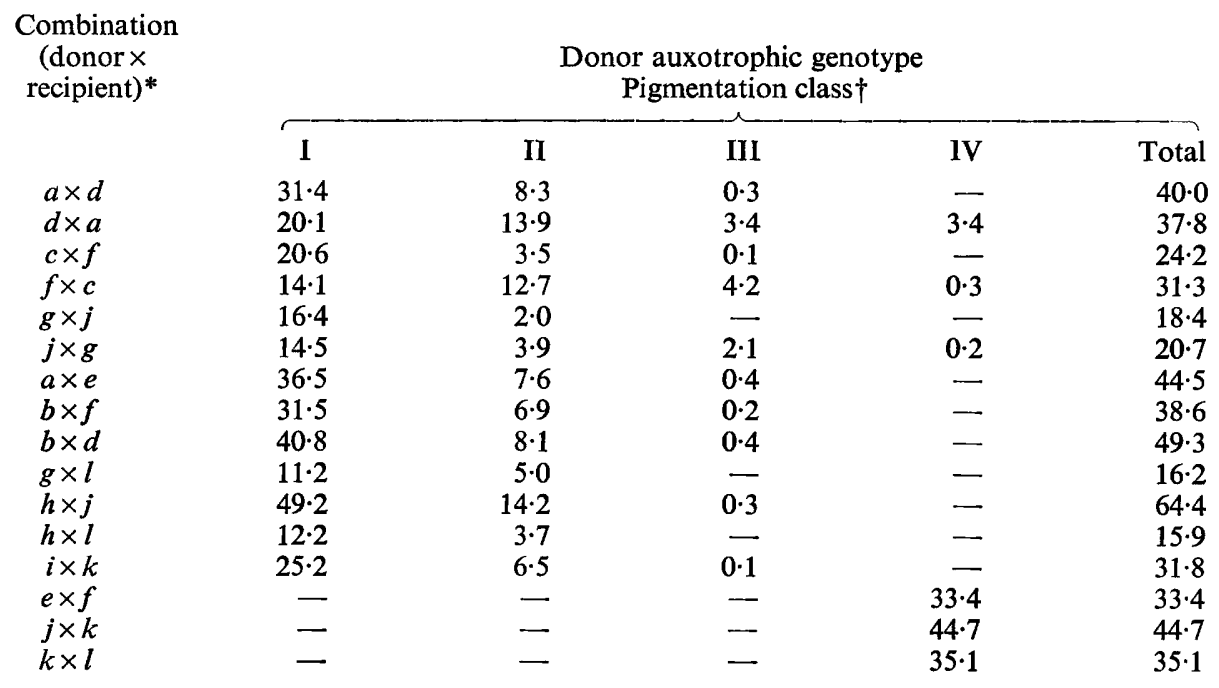

Recipient auxotrophic genotype

Pigmentation class $\dagger$

\begin{tabular}{|c|c|c|c|c|c|}
\hline & I & II & III & IV & Total \\
\hline$a \times d$ & 37.8 & 14.5 & 6.7 & 1.0 & 60.0 \\
\hline$d \times a$ & $40 \cdot 8$ & $21 \cdot 4$ & - & - & $62 \cdot 2$ \\
\hline$c \times f$ & $51 \cdot 6$ & $17 \cdot 1$ & $6 \cdot 3$ & 0.8 & $75 \cdot 8$ \\
\hline$f \times c$ & $48 \cdot 1$ & 20.5 & $0 \cdot 1$ & - & $68 \cdot 7$ \\
\hline$g \times j$ & $48 \cdot 0$ & $24 \cdot 6$ & $7 \cdot 1$ & 0.9 & $81 \cdot 6$ \\
\hline$j \times g$ & $55 \cdot 4$ & $23 \cdot 7$ & 0.2 & - & $79 \cdot 3$ \\
\hline$a \times e$ & $30 \cdot 1$ & $19 \cdot 0$ & $5 \cdot 3$ & $1 \cdot 1$ & 55.5 \\
\hline$b \times f$ & $32 \cdot 9$ & $20 \cdot 4$ & $7 \cdot 1$ & 1.0 & $61 \cdot 4$ \\
\hline$b \times d$ & 26.9 & $17 \cdot 2$ & 6.0 & 0.6 & 5 \\
\hline$g \times l$ & $46 \cdot 2$ & 28.9 & $7 \cdot 6$ & $1 \cdot 1$ & $83 \cdot 8$ \\
\hline$h \times j$ & $19 \cdot 7$ & $11 \cdot 1$ & $4 \cdot 3$ & 0.5 & $35 \cdot 6$ \\
\hline$h \times l$ & $51 \cdot 6$ & 23.8 & $7 \cdot 5$ & 1.2 & $84 \cdot 1$ \\
\hline$i \times k$ & $41 \cdot 9$ & $19 \cdot 6$ & $5 \cdot 8$ & 0.9 & 68 \\
\hline$e \times f$ & - & - & - & $66 \cdot 6$ & $66 . \overline{6}$ \\
\hline$j \times k$ & - & - & - & $55 \cdot 3$ & 55. \\
\hline$k \times l$ & - & - & - & 64.9 & 6 \\
\hline
\end{tabular}

* For strain phenotypes and genotypes, see Table 1.

$\uparrow$ Pigmentation classes: I, wild-type pigmentation; II, almost wild-type pigmentation; III, partially hyaline appearance (few dots of pigmentation in the centre of colonies); IV, completely hyaline (no pigment).

\section{Micro-injection of nuclei}

In preliminary experiments only 2.5 to $10.0 \%$ of the injected cells survived, but with improvements of the technique survival reached 30 to $40 \%$. However, this frequency was much lower than that obtained for protoplasmic injections $(80 \%)$. When spores from colonies formed by the surviving recipient cells were analysed, only recipient parental 
Table 5. Analysis of conidia from the entire surface of Verticillium colonies resulting from protoplasmic transplantation where nuclear transfer (prototrophy) failed to occur

Conidia were classified according to their phenotypes with respect to darkly-pigmented resting structures, and the results show the percentages in each pigmentation class. At least 400 conidia were tested for each heterokaryon.

\begin{tabular}{|c|c|c|c|c|}
\hline \multirow{2}{*}{$\begin{array}{l}\text { Combination } \\
\text { (donor } \times \\
\text { recipient)* }\end{array}$} & \multicolumn{4}{|c|}{ Pigmentation class $\uparrow$} \\
\hline & I & II & III & IV \\
\hline$a \times d$ & $52 \cdot 4$ & $37 \cdot 4$ & $8 \cdot 3$ & 1.9 \\
\hline$d \times a$ & $56 \cdot 5$ & $40 \cdot 1$ & $3 \cdot 4$ & - \\
\hline$c \times f$ & $58 \cdot 7$ & $32 \cdot 7$ & $8 \cdot 0$ & $1 \cdot 6$ \\
\hline$f \times c$ & $54 \cdot 7$ & $40 \cdot 5$ & $4 \cdot 8$ & - \\
\hline$g \times j$ & $47 \cdot 3$ & $39 \cdot 7$ & $11 \cdot 2$ & $1 \cdot 8$ \\
\hline$j \times g$ & $65 \cdot 5$ & $30 \cdot 6$ & 3.9 & 一 \\
\hline$a \times e$ & $44 \cdot 9$ & $36 \cdot 6$ & 16.0 & $2 \cdot 5$ \\
\hline$b \times f$ & $49 \cdot 6$ & $37 \cdot 1$ & $11 \cdot 6$ & $1 \cdot 7$ \\
\hline$b \times d$ & $59 \cdot 7$ & $29 \cdot 3$ & $9 \cdot 5$ & $1 \cdot 5$ \\
\hline$g \times l$ & $40 \cdot 0$ & $40 \cdot 9$ & $16 \cdot 4$ & $2 \cdot 7$ \\
\hline$h \times j$ & $53 \cdot 4$ & $34 \cdot 4$ & $11 \cdot 2$ & 1.0 \\
\hline$h \times l$ & $46 \cdot 1$ & $38 \cdot 1$ & $12 \cdot 8$ & 3.0 \\
\hline$i \times k$ & $47 \cdot 9$ & $37 \cdot 6$ & $12 \cdot 2$ & $2 \cdot 3$ \\
\hline$e \times f$ & 一 & 一 & - & $100 \cdot 0$ \\
\hline$j \times k$ & - & - & - & $100 \cdot 0$ \\
\hline$k \times l$ & 一 & - & - & $100 \cdot 0$ \\
\hline
\end{tabular}

auxotroph genotypes were recovered. Only in two cases were some darkly-pigmented $(0.1 \%$ and $0.3 \%$, respectively) colonies recovered from $\mathrm{Hyl}$ recipients injected with nuclei derived from $\mathrm{Hyl}^{+}$auxotrophic strains; in one other case some hyaline $(0.1 \%)$ colonies were recovered from $\mathrm{Hyl}^{+}$recipients injected with $\mathrm{Hyl}$ nuclei. Because 'artificial' heterokaryons were not produced using this method, it is concluded that donor nuclei apparently did not survive.

\section{Micro-injection of mitochondrial preparations}

The changes in behaviour and morphology brought about by micro-injection of mitochondrial preparations varied greatly. In general, colonies arising from injected recipients had a variable growth form and slow rate of growth. Conidia from micro-injected cultures often formed prostrate hyphae with multiple branches composed of short, thick, septate cells when compared with both 'natural' and 'artificial' heterokaryons produced by the above methods. The conidiophores were short, thick, and not always typically verticillate. The phialides bore few conidia and yields rarely exceeded $3 \times 10^{4}$ spores per plate in 5 week-old cultures. After two spore generations, however, sporulation usually reached normal parental levels.

The frequency of survivors amongst recipient cells injected with mitochondrial preparations was lower $(40$ to $45 \%$ ) than that obtained from protoplasmic injections $(80 \%)$. Conidia from colonies arising from successfully injected cells were analysed and the results are shown in Table 6. When mitochondrial fractions of darkly-pigmented $\left(\mathrm{Hyl}^{+} \mathrm{Sot}^{+}\right.$ or $\mathrm{Hyl}^{+} \mathrm{Sot}$ ) auxotrophic strains were injected into darkly-pigmented $\left(\mathrm{Hyl}^{+} \mathrm{Sot}^{+} \mathrm{Or} \mathrm{Hyl}^{+} \mathrm{Sot}\right)$ recipients, most of the colonies recovered ( 88 to $89 \%$ ) were darkly-pigmented, but small numbers of partially hyaline (10 to $11 \%)$ and completely hyaline $(0.3$ to $4 \%)$ colonies were also observed.

Conidial analysis of spores recovered from cultures of $\mathrm{Hyl}$ recipients injected with $\mathrm{Hyl}^{+}$ or $\mathrm{Hyl}^{+}$Sot mitochondrial fractions resulted in colonies with variable morphology. Most of these colonies were hyaline ( 78 to $87 \%$ ), but all the other types were also recovered, i.e. 
Table 6. Analysis of conidia from the entire surface of Verticillium colonies resulting from successful mitochondrial transplantation

Conidia were classified according to their phenotypes with respect to darkly-pigmented resting structures, and the results show the percentages in each pigmentation class. At least 400 conidia were tested for each strain. Only recipient auxotrophic parental genotypes were recovered.

\begin{tabular}{|c|c|c|c|c|}
\hline \multirow{2}{*}{$\begin{array}{c}\text { Combination } \\
\text { (donor } \times \\
\text { recipient })^{*}\end{array}$} & \multicolumn{4}{|c|}{ Pigmentation class $\uparrow$} \\
\hline & I & II & III & IV \\
\hline$a \times e$ & $1 \cdot 2$ & $2 \cdot 8$ & $11 \cdot 5$ & $84 \cdot 5$ \\
\hline$e \times a$ & $47 \cdot 4$ & $37 \cdot 6$ & 14.6 & 0.4 \\
\hline$g \times i$ & 52.5 & $36 \cdot 7$ & $10 \cdot 5$ & 0.3 \\
\hline$a \times d$ & $1 \cdot 4$ & $2 \cdot 3$ & $12 \cdot 7$ & $83 \cdot 6$ \\
\hline$b \times f$ & 1.0 & $1 \cdot 7$ & $10 \cdot 3$ & $87 \cdot 0$ \\
\hline$g \times j$ & $1 \cdot 7$ & $2 \cdot 5$ & 11.9 & 83.9 \\
\hline$i \times l$ & $2 \cdot 2$ & $3 \cdot 1$ & $15 \cdot 9$ & $78 \cdot 8$ \\
\hline$e \times f$ & $2 \cdot 0$ & $3 \cdot 3$ & $15 \cdot 9$ & $78 \cdot 8$ \\
\hline$b \times a$ & $53 \cdot 4$ & $35 \cdot 0$ & $11 \cdot 2$ & 0.4 \\
\hline
\end{tabular}

partially hyaline colonies (10 to $15 \%$ ), and wild-type and near wild-type ( 1 to $2 \%$ ) colonies. Results even from replicate experiments varied greatly (e.g. $a \times e$ from four different successful micro-injected recipients resulted in $0 \cdot 0,0 \cdot 3,3 \cdot 0$ and $1 \cdot 5 \%$ for wild-type; $0 \cdot 4,0.9,5.4$ and $4.5 \%$ for near wild-type; $16 \cdot 3,13 \cdot 2,6 \cdot 0$ and $10 \cdot 5 \%$ for partially hyaline and $83 \cdot 3,85 \cdot 6,85 \cdot 6$ and $83.3 \%$ for hyaline, respectively), and so also did those from the next generation of spores. It was only after the third spore generation that colonies regained stable growth rates and levels of pigmentation. The percentage of colonies recovered from Hyl auxotrophic recipients injected with $\mathrm{Hyl}^{+}$mitochondrial preparations which had at least some darklypigmented resting structures was higher $(13.0$ to $21.2 \%)$ than that obtained from injections with nuclear preparations $(0 \cdot 3$ to $0.5 \%)$. This suggests that one or more factors controlling darkly-pigmented structures were contained in the mitochondrial preparations from the $\mathrm{Hyl}^{+}$donor cytoplasm.

\section{DISCUSSION}

The results indicate that preparations of protoplasm, nuclei and mitochondria can be introduced into living Verticillium cells, although the failure of nuclear transfers needs further study. The injection of a cell-free, compatible protoplasm produced no obvious damage and $80 \%$ of the hyphae survived. The analyses of conidial genotypes formed by 'artificial' heterokaryons showed that viable nuclei could be injected with protoplasmic fluid; $21 \%$ of the injected recipient cells originally classified as containing nuclei of only the recipient cell later yielded a small proportion with donor types. Conidial analyses of 'artificial' heterokaryons arising from reciprocal injection of $\mathrm{Hyl}^{+}$and $\mathrm{Hyl}$ auxotrophic protoplasm into $\mathrm{Hyl}$ and $\mathrm{Hyl}^{+}$complementary auxotrophic recipients, respectively, resulted in recovery frequencies of all biochemical markers that confirmed a nuclear pattern of inheritance as already demonstrated in 'natural' heterokaryon conidial analyses (Typas \& Heale, 1976b). Most morphological phenotypes recovered from reciprocal injections of protoplasm were more or less darkly-pigmented (92 to $96 \%$ ) and only a few were partially hyaline $(0.1$ to $7.0 \%)$ or completely hyaline $(0.0$ to $1.0 \%)$. This confirms the observations on 'natural' heterokaryons and heterozygous diploid analyses (Typas \& Heale, 1978) that a major factor controlling production of darkly-pigmented resting structures in the fungus is cytoplasmic rather than nuclear. The fact that similar results were obtained from reciprocal injections shows that the cytoplasmic factor is found in an active form only in $\mathrm{Hyl}^{+}$cytoplasm, and is either completely absent or inactive in the Hyl cytoplasm. Further 
evidence came from the analyses of 'artificial' heterokaryons formed by $\mathrm{Hyl} \mathrm{Sot}{ }^{+}$or $\mathrm{Hyl}$ recipient cells injected with $\mathrm{Hyl}^{+}$and $\mathrm{Hyl}^{+}$Sot protoplasm, respectively. Here the different patterns of inheritance, i.e. cytoplasmic for Hyl and nuclear for Sot, are clearly established.

Conidial analyses of injected $\mathrm{Hyl}$ recipients with $\mathrm{Hyl}^{+}$protoplasm, which resulted in the final recovery of only recipient auxotrophic genotypes, indicated the failure of viable nuclear transfer into the host cell. Most recipients treated in this way were therefore heteroplasmons and contained wild-type $\left(\mathrm{Hyl}^{+}\right)$and mutant $(\mathrm{Hyl})$ homologues of a cytoplasmic determinant. The recovery of 80 to $97 \%$ darkly-pigmented phenotypes from conidia produced by the heteroplasmons is good evidence for the cytoplasmic nature of the Hyl marker. The few partially hyaline (4 to $16 \%$ ) and completely hyaline (1.0 to $3.0 \%$ ) phenotypes, which were also isolated from heteroplasmon analyses, can be attributed to (i) the absence of the cytoplasmic determinant from the portion of cytoplasm injected into the host cell, (ii) failure of the cytoplasmic determinant to migrate into the daughter conidium following a nuclear division, or (iii) some nuclear involvement in the inheritance of the factor (e.g. regulation).

The slower growth rate of 'artificial' heterokaryons compared with controls (punctured and uninjected or punctured and injected with sucrose) and 'natural' heterokaryons may be due to an imbalance in the initial nuclear proportions in the injected cell, as also shown in Neurospora (Wilson et al., 1961; Wilson, 1963).

The survival of recipient cells injected with preparations of nuclei was lower (30 to $40 \%$ ) than that of protoplasmic injections $(80 \%)$. Such a difference suggests that a substance is being produced by the donor or treated recipient nuclei, or is introduced in the host cells with the nuclear preparation, which results in lethal damage in the injected cell. This substance may operate in a similar manner to the lethal incompatibility in Neurospora (Wilson, 1963 ) or to that found for incompatible mating types which results in the death of the recipient cell (Garnjobst \& Wilson, 1956). The results of mitochondrial injections are of interest because of their possible role in transmitting the cytoplasmic factor ( $\mathrm{Hyl}$ ) controlling dark resting structures in Verticillium. The higher frequencies (13 to $21 \%$ ) at which $\mathrm{Hyl}^{+}$ and partially hyaline colonies were recovered from $\mathrm{Hyl}$ auxotrophic recipients injected with $\mathrm{Hyl}^{+}$mitochondrial preparations compared with that from the corresponding nuclear preparations $(0.3$ to $0.5 \%)$ suggests that one or more factors controlling darkly-pigmented resting structures was present in the mitochondrial preparation from the $\mathrm{Hyl}^{+}$donor cytoplasm. The present evidence does not reveal the exact nature of the cytoplasmic factor concerned with the inheritance of dark resting structures in Verticillium. Nevertheless, there is some indication that this extrachromosomal particle is somehow associated with mitochondria or with mitochondrial preparations, and this possibility is now being investigated.

We thank Dr J. L. MacGregor, University of Lyon, France for his helpful technical assistance, advice and criticism during these experiments.

\section{REFERENCES}

BARBER, M. A. (1911). The effect on the protoplasm of Nitella of various chemical substances and of microorganisms introduced into the cavity of the living cell. Journal of Infectious Diseases 9, 117129.

BrandT, W. H. \& Roth, J. N. (1965). Loss of melanin-containing structures by Verticillium species. Phytopathology 55, 1200-1202.

Briggs, R. \& KING, T. J. (1959). Nucleocytoplasmic interactions in egg and embryos. In The Cell, vol. 1, pp. 537-617. Edited by J. Brachet \& A. E. Mirsky. New York: Academic Press.
BURGEFF, E. W. (1914). Untersuchungen über Variabilität, Sexualität und Erblichkeit bei Phycomyces nitens. Flora, Jena 107, 259316.

Comandon, J. \& de Fonbrune, P. (1939). Greffe nucléaire totale, simple ou multiple, chez une amibe. Comptes rendus des séances de la Société de biologie 130, 744-748.

Diakumacos, E. G., Garnjobst, L. \& Tatum, E. L. (1965). A cytoplasmic character in Neurospora crassa. The role of nuclei and mitochondria. Journal of Cell Biology 26, 427-443. 
Garnjobst, L. \& Wilson, J. F. (1956). Heterocaryosis and protoplasmic incompatibility in Neurospora crassa. Proceedings of the National Academy of Sciences of the United States of America 42, 613-618.

GibsoN, I. (1973). Transplantation of killer endosymbionts in Paramecium. Nature, London 241, 127-129.

Hansen, H. N. (1938). The dual phenomenon in imperfect fungi. Mycologia 30, 442-455.

Hastie, A. C. (1964). The parasexual cycle in Verticillium albo-atrum. Genetical Research 5, 305-315

Heale, J. B. \& IsAaC, I. (1964). Dark pigment formation in Verticillium albo-atrum. Nature, London 202, 412-413.

Holwill, M. E. J. \& McGregor, J. L. (1974). Micromanipulation of the flagellum of Crithidia oncopelti. I. Mechanical effects. Journal of Experimental Biology 60, 437-444.

IsAAC, I. (1949). A comparative study of pathogenic isolates of Verticillium. Transactions of the British Mycological Society 32, 137-157.

JINKS, J. L. (1964). In Extrachromosomal Inheritance, pp. 1-192. Edited by S. R. Suskind \& P. E. Hartman. New Jersey: Prentice-Hall.

KNowles, J. K. C. (1974). An improved microinjection technique in Paramecium aurelia. Transfer of mitochondria conferring erythromycinresistance. Experimental Cell Research 88, 7987.

KoIzum, S. (1974). Microinjection and transfer of cytoplasm in Paramecium. Experiments on the transfer of Kappa particles into cells at different stages. Experimental Cell Research 88, 74-78.

KopaC, M. J. (1959). Micrurgical studies on living cells. In The Cell, vol. 1, pp. 161-191. Edited by J. Brachet \& A. E. Mirsky. New York: Academic Press.

Luck, D. J. L. (1963). Formation of mitochondria in Neurospora crassa. A quantitative radioautographic study. Journal of Cell Biology 16, 483499.

PegG, G. F. (1957). A hyaline variant of Verticillium albo-atrum pathogenic to tomato plants. Phytopathology 47, 57-58.
REICH, E. \& TsUdA, S. (1961). Isolation of nuclei of Neurospora crassa. Biochimica et biophysica acta 53, 574-575.

Robinson, D. B., Larson, R. H. \& Walker, J. C. (1957). Verticillium wilt of potato in relation to symptoms, epidemiology and variability of the pathogen. Wisconsin Agricultural Experimental Station Research Bulletin 202, 1-49.

Tolmsoff, W. J. (1972). Diploidization and heritable gene repression-derepression as major sources for variability in morphology, metabolism, and pathogenicity in Verticillium species. Phytopathology 62, 407-413.

Tolmsoff, W. J. (1973). Life cycles of Verticillium species. In Verticillium wilt of cotton, Proceedings of a Work Conference, National Cotton Pathology Research Laboratory, College Station, Texas, 1971 (ARS-S-19), pp. 20-38.

TyPas, M. A. (1976). Genetical aspects of the mechanisms of variation and differentiation in species of Verticillium. Ph.D. thesis, London University.

Typas, M. A. \& Heale, J. B. (1976a). Acriflavineinduced hyaline variants of Verticillium albo-atrum and $V$. dahliae. Transactions of the British Mycological society 66, 15-25.

Typas, M. A. \& Heale, J. B. (1976b). Heterokaryosis and the rôle of cytoplasmic inheritance in dark resting structure formation in Verticillium spp. Molecular and General Genetics 146, 17-26.

Typas, M. A. \& Heale, J. B. (1978). Heterozygous diploid analyses via the parasexual cycle and a cytoplasmic pattern of inheritance in Verticillium spp. Genetical Research 31, 131-144.

WiLson, J.F. (1961). Micrurgical techniques for Neurospora. American Journal of Botany 48, 46-51.

WILsoN, J. F. (1963). Transplantation of nuclei in Neurospora crassa. American Journal of Botany 50, 780-786.

WILSON, J. F. (1969). Mitochondrial transplantation studies in Neurospora. Proceedings of the XI International Botanical Congress, Seattle 1, 240.

Wilson, J. F., Garnjobst, L. \& Tatum, E. L. (1961). Heterocaryon incompatibility in Neurospora crassa. Microinjection studies. American Journal of Botany 48, 299-305. 